\title{
Preparation and characterization of norcantharidin liposomes modified with stearyl glycyrrhetinate
}

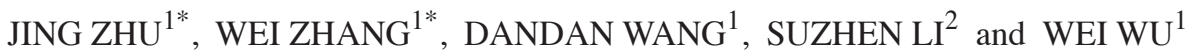 \\ ${ }^{1}$ Department of Pharmaceutics, College of Pharmacy, Guilin Medical University; ${ }^{2}$ Clinical Experimental \\ Teaching Center, Affiliated Hospital of Guilin Medical University, Guilin, Guangxi 541004, P.R. China
}

Received November 4, 2017; Accepted April 13, 2018

DOI: $10.3892 /$ etm.2018.6416

\begin{abstract}
In the current study, norcantharidin (NCTD)-loaded liposomes (LIPs) modified with stearyl glycyrrhetinate (SG; SG-NCTD-LIP) were prepared by the ethanol injection method. To increase the drug encapsulation efficiency (EE), the formulation of NCTD-LIP was optimized by single factor test and orthogonal design. The release of NCTD in vitro from SG-NCTD-LIP was evaluated by equilibrium dialysis. The cytotoxicity of SG-NCTD-LIP in HepG2 was investigated by MTT assay. The results revealed that the EE of liposomes was $\sim 27.80 \%$, the average SG-NCTD-LIP was $87.5 \mathrm{~nm}$, the in vitro NCTD release from SG-NCTD-LIP was delayed compared with NCTD in solution and the drug-release kinetic followed a first-order model. MTT assays revealed increased cytotoxicity activity against HepG2 cells for SG-NCTD-LIP compared with free NCTD. In conclusion, SG-NCTD-LIP prepared in the present study may be a promising liposomal drug delivery system for anticancer drugs in liver-targeting therapy.
\end{abstract}

\section{Introduction}

Norcantharidin (NCTD; Fig. 1), the demethylated derivative of cantharidin obtained from the dried body of the Chinese blister beetle (Mylabris spp.) (1), has been used as an anticancer drug in China $(2,3)$. NCTD acts by inducing cell death through mechanisms involving the response to DNA damage and apoptosis by activation of the protein kinase $\mathrm{C}$ signaling pathway (4). Like sulfonamides for carbonic anhydrase or hydroxamic acids for metalloproteinases, NCTD describes the archetypal small molecule protein phosphatase inhibitor (5) and has been used to inhibit proliferation and metastasis of

Correspondence to: Professor Wei Wu, Department of Pharmaceutics, College of Pharmacy, Guilin Medical University, 109 Huancheng Road, Guilin, Guangxi 541004, P.R. China

E-mail: wuweigx@126.com

${ }^{*}$ Contributed equally

Key words: liposomes, norcantharidin, stearyl glycyrrhetinate, drug delivery system, liver-targeting multiple types of carcinoma (6,7). Previous studies indicated that NCTD has therapeutic value in the treatment of various types of cancer, including liver cancer, when administered orally or intravenously $(6,8-11)$. However, the application of NCTD is limited by numerous factors, including short half-life, high systemic toxicity, high incidence of adverse effects and poor bioavailability in the physiological environment $(12,13)$. To improve the safety and efficacy of NCTD, NCTD nanoscale drug delivery systems (DDS) have been studied (14-16).

Liposomes are spheres with a lipid bilayer shell prepared with a variety of phospholipids, which have been extensively studied since the 1960s (17-19). Cholesterol is an additional compound in the liposomal structure that could regulate the fluidity of phospholipid membrane and may control the retention of drugs (20). Therefore, liposomes may be used as nanoscale vehicles for the administration of drugs. With good biocompatibility and low toxicity, liposomes have been revealed to enhance the therapeutic activity of numerous anticancer drugs (21-23). However, conventional liposomes have certain disadvantages, including the uptake by the reticuloendothelial system (RES) $(24,25)$, the lack of tumor-specificity and insufficient uptake at tumor sites. Ligand-targeted liposomes, where specific ligands are used to modify liposomes, have demonstrated the potential to increase therapeutic efficacy and reduce adverse effects through the interaction between a specific ligand and the target molecule, and facilitating the receptor mediated endocytosis of liposomes (26).

Glycyrrhetinic acid (GA) may be obtained by hydrolysis of glycyrrhicinate, extracted from the roots of liquorice (Glycyrrhiza glabra) (27). GA possesses many pharmacological and biological relevant activities, including anti-inflammatory, antiallergic, antiulcer, antioxidant, antihepatotoxic, antineoplasmic and antiviral activities (28-30). Studies have reported that specific GA binding sites may be located on the cell membrane of hepatocytes $(31,32)$. GA and its derivatives may be used as ligands targeting the liver $(33,34)$. Stearyl glycyrrhetinate (SG; Fig. 2), the stearyl ester of 18 - $\beta$-glycyrrhetinic acid, has been demonstrated to improve antiviral effects, reduce inflammation and suppress allergies in the pharmaceutical and cosmetic industry $(35,36)$. Compared with GA, SG has an increased compatibility as its hydrophobic tail may be adsorbed into the lipid layer of liposomes while exposing the hydrophilic GA moiety at the surface.

The objective of the current study was to prepare NCTD-loaded liposomes modified with SG (SG-NCTD-LIP) 
by ethanol injection. Single factor test and orthogonal design were used to optimize the formulation of SG-NCTD-LIP. The characterization of the prepared liposomes included physical morphology, particle size and encapsulation efficiency (EE). Equilibrium dialysis was used to investigate in vitro release characteristics of SG-NCTD-LIP. Furthermore, in vitro cytotoxicity of SG-NCTD-LIP in HepG2 cells was determined by MTT assay.

\section{Materials and methods}

Materials. NCTD (>99\%) was purchased from Sunray Pharmaceutical Co., Ltd. (Suzhou, China). SG (>98\%) was obtained from Xi'an Realin Biotechnology Co., Ltd. (Xi'an, China). Egg phosphatidylcholine (EPC) was supplied by Lipoid GmbH (Ludwigshafen, Germany). Cholesterol was purchased from Beijing Solarbio Science \& Technology Co., Ltd. (Beijing, China). HPLC-grade acetonitrile was purchased from Sigma-Aldrich (Merck KGaA, Darmstadt, Germany). All other chemicals used in this study were of analytical grade.

Preparation of SG-NCTD-LIP. Liposomes were prepared by ethanol injection method (Fig. 3) (37,38). EPC (0.1-0.4\%), cholesterol (0.03-0.20\%) and SG (0.04\%) were dissolved in $2 \mathrm{ml}$ absolute ethanol. The aqueous phase was prepared by dissolving NCTD (0.02-0.08\%) in $15 \mathrm{ml} \mathrm{PBS} \mathrm{(pH} \mathrm{7.0)} \mathrm{and}$ heating in a water bath at $37^{\circ} \mathrm{C}$. The $2 \mathrm{ml}$ ethanol phase was immediately injected into the heated aqueous solution through a fine needle under magnetic stirring. The liposome dispersions were incubated at between $50^{\circ} \mathrm{C}$ for $30 \mathrm{~min}$ with gentle stirring.

Determination of NCTD content and EE. SG-NCTD-LIP was separated from the free drug by equilibrium dialysis (the dialysis membrane bag molecular weight cut-off of 8,000-14,000). The liposome fraction was obtained and the liposomes were ruptured in methanol for drug solubilisation. The resulting solution was sonicated $\left(150 \mathrm{~W}\right.$ at $\left.25^{\circ} \mathrm{C}\right)$ for $5 \mathrm{~min}$ and filtered through polytetrafluorethylene membranes $(0.22 \mu \mathrm{m})$. The drug concentration was determined using a reversed-phase high-performance liquid chromatography (RP-HPLC) system (LC-20AT; Shimadzu Corporation, Kyoto, Japan) at $25^{\circ} \mathrm{C}$ and $98 \%$ NCTD was used as a quantification standard. The mobile phase was acetonitrile/water (10:90, $\mathrm{v} / \mathrm{v} ; \mathrm{pH} 3.1)$ and an isocratic elution was performed using a WondaCract ODS-2 column $(5 \mu \mathrm{m}, 4.6 \times 250 \mathrm{~mm}$; Shimadzu Corporation) with a flow rate of $0.8 \mathrm{ml} / \mathrm{min}$. NCTD was detected at $220 \mathrm{~nm}$. The EE was determined by dividing the amount of drug in the liposome fraction by the amount of drug in the total fractions.

Optimization of SG-NCTD-LIP formulation. Using the ethanol injection method, several factors were trialed to achieve optimal formulation, including NCTD-phospholipid mass ratio (factor A), phospholipid concentration (factor B), incubation temperature (factor $\mathrm{C}$ ) and cholesterol-phospholipid mass ratio (factor $\mathrm{D}$ ) during the fabrication process. Only one factor was replaced in each series of experiments. When changing the amount of phospholipids, the NCTD-phospholipid mass ratio was 1:5, cholesterol-phospholipid mass ratio was 1:7

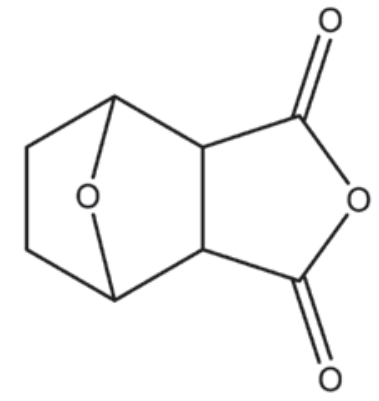

Figure 1. Chemical structure of norcantharidin.

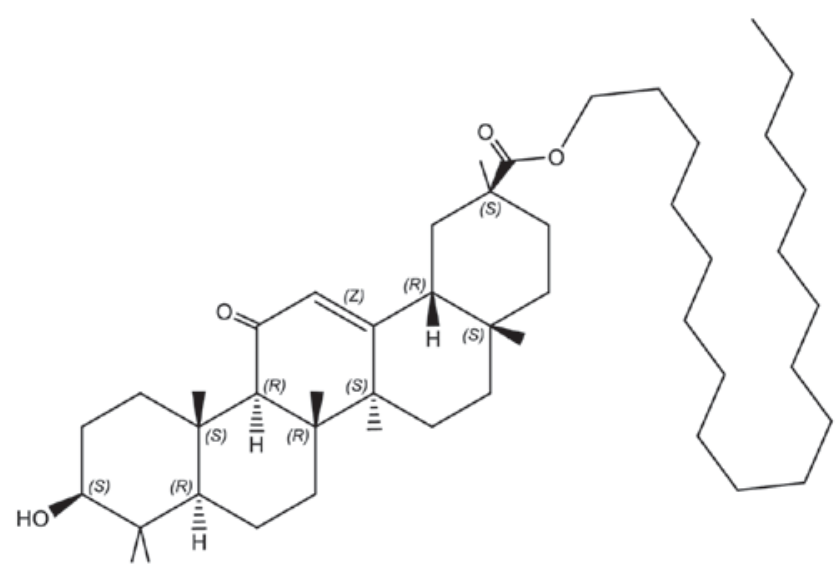

Figure 2. Chemical structure of stearyl glycyrrhetinate.

and incubation temperature was $50^{\circ} \mathrm{C}$. When changing the amount of NCTD, the phospholipid concentration was $0.24 \%$, cholesterol-phospholipid mass ratio was $1: 7$ and incubation temperature was $50^{\circ} \mathrm{C}$. When changing cholesterol-phospholipid mass ratio, the phospholipid concentration was $0.24 \%$, NCTD-phospholipid mass ratio was 1:20 and incubation temperature was $50^{\circ} \mathrm{C}$. When changing incubation temperature, the phospholipid concentration was $0.24 \%$, NCTD-phospholipid mass ratio was 1:20 and cholesterol-phospholipid mass ratio was 1:5. Based on the investigation of factors, the four aforementioned factors were selected, and three levels of each factor were designated for the orthogonal design, with the $\mathrm{EE}$ as the investigating indicator to screen the formulation.

SG-NCTD-LIP size and polydispersity index (PDI). The particle size and PDI of SG-NCTD-LIP were determined at $25^{\circ} \mathrm{C}$ by dynamic light scattering (Nano-ZS; Malvern Instruments, Ltd., Malvern, UK). Prior to measurement, the liposome dispersions were diluted 10 times with distilled water. Measurements were performed in triplicate on independent formulations at a detection angle of $90^{\circ}$.

Transmission electron microscopy (TEM) measurement of SG-NCTD-LIP. A diluted SG-NCTD-LIP sample $(0.018 \mathrm{mg} / \mathrm{ml} ; 10 \mu \mathrm{l})$ was placed on a copper grid and air-dried at room temperature. Subsequently, a drop of $1 \%(\mathrm{w} / \mathrm{v})$ aqueous solution of phosphotungstic acid was added for negative staining; the sample was dried at room temperature for $20 \mathrm{~min}$. The analyses of the vesicle shape were carried out on 


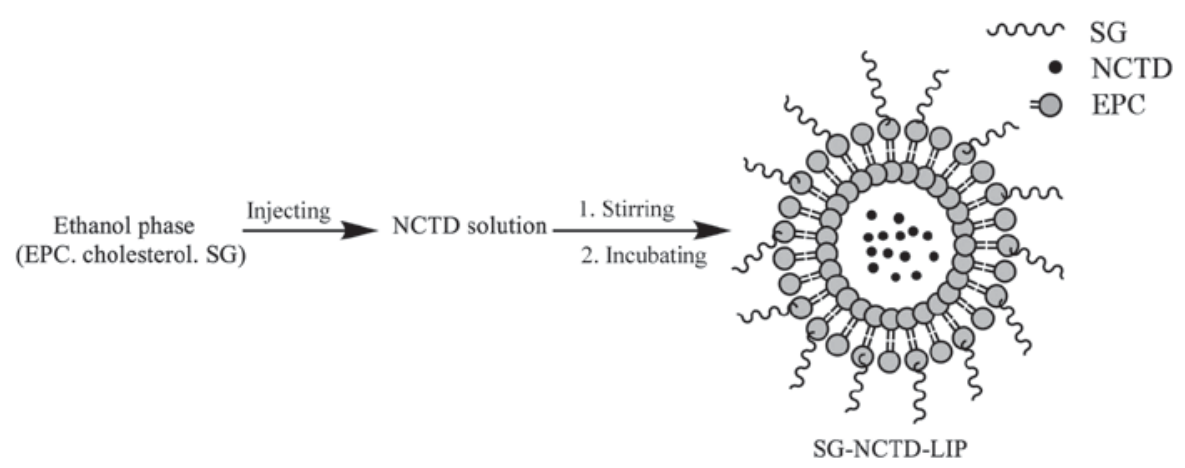

Figure 3. Ethanol injection method for preparation of SG-NCTD-LIP. The ethanol phase, containing EPC, cholesterol and SG in ethanol, was injected into the aqueous phase, containing NCTD dissolved in PBS, and incubated stirring for 30 min to yield SG-NCTD-LIP. EPC, egg phosphatidylcholine; SG, stearyl glycyrrhetinate; NCTD, norcantharidin; SG-NCTD-LIP, NCTD-loaded liposomes modified with SG.

a JEM-2100F transmission electron microscope (JEOL, Ltd., Tokyo, Japan) with an acceleration of $150 \mathrm{kV}$.

SG-NCTD-LIP stability studies. SG-NCTD-LIP suspension stability was assessed over 3 months of storage at $4^{\circ} \mathrm{C}$ by means of particle size, PDI and EE measurements.

In vitro drug release study. In vitro drug release was performed as described previously (39). Dialysis tubing (molecular weight cut off, 8,000-14,000 $\mathrm{g} / \mathrm{mol}$ ) was incubated in distilled water for $12 \mathrm{~h}$ at room temperature. SG-NCTD-LIP $(0.18 \mathrm{mg} / \mathrm{ml} .8 \mathrm{ml})$ and NCTD solution $(0.18 \mathrm{mg} / \mathrm{ml}, 8 \mathrm{ml})$ were placed in separate dialysis tubing and each incubated with stirring in $80 \mathrm{ml} \mathrm{PBS} \mathrm{(pH} \mathrm{7.4,} \mathrm{release} \mathrm{medium)} \mathrm{at} 37^{\circ} \mathrm{C}$. Following $0.25,0.5,1,1.5,2,4,8$ and $12 \mathrm{~h}$ incubation, $0.5 \mathrm{ml}$ release medium was collected and replaced with an equal volume of fresh medium. NCTD concentration was analyzed by RP-HPLC.

Cell culture. HepG2, an immortalized cell line consisting of hepatoblastoma cells, was obtained from the Laboratory of Hepatobiliary and Pancreatic Surgery (Affiliated Hospital of Guilin Medical University, Guilin, China). Cells were cultured in Dulbecco's modified Eagle's medium supplemented with $10 \%$ fetal bovine serum (FBS; both Gibco; Thermo Fisher Scientific, Inc., Waltham, MA, USA), $100 \mathrm{U} / \mathrm{ml}$ penicillin and $100 \mu \mathrm{g} / \mathrm{ml}$ streptomycin under $5 \% \mathrm{CO}_{2}$ atmosphere at $37^{\circ} \mathrm{C}$ for $24 \mathrm{~h}$.

Cytotoxicity study. 3-(4,5-dimethylthiazol-2-yl)-2,5-diphenyltetrazolium bromide (MTT) assays were used to study the proliferative effect of SG-NCTD-LIP. HepG2 cells were plated in 96-well culture plates at 4,000 cells/well. After overnight incubation at $37^{\circ} \mathrm{C}$, cells were treated with varying concentrations $(2.5,5,10,20$ and $40 \mu \mathrm{g} / \mathrm{ml})$ of SG-modified blank liposomes (SG-LIP), NCTD solution, liposomes loaded with NCTD (NCTD-LIP) or SG-NCTD-LIP and were incubated for $48 \mathrm{~h}$ at $37^{\circ} \mathrm{C}$. MTT (Sigma-Aldrich; Merck KGaA) solution $(20 \mu \mathrm{l} ; 5 \mathrm{mg} / \mathrm{ml})$ was added to each well. Following $4 \mathrm{~h}$ incubation at $37^{\circ} \mathrm{C}$, the medium was replaced with $150 \mu$ l dimethyl sulfoxide to dissolve formazan crystals. Optical densities (ODs) were measured at $490 \mathrm{~nm}$ with an ELISA reader. The half-maximal inhibitory concentration $\left(\mathrm{IC}_{50}\right)$ was calculated using SPSS (version 22.0; IBM Corp., Armonk, NY, USA). The inhibition ratio (IR) was calculated as follows: IR=1-OD of cells treated with sample/OD of culture medium.

Statistical analysis. Data were analyzed using SPSS software (version 22.0; IBM Corp., Armonk, NY, USA) and results are expressed as mean \pm standard deviation. One-way analysis of variance followed by a Fisher's Least Significant Difference post-hoc test was used to assess the significance of the differences among various groups. Correlation coefficient (R) is a variable that demonstrated the degree of linear correlation between variables. $\mathrm{P}<0.05$ was considered to indicate a statistically significant difference.

\section{Results and Discussion}

Effect of different preparation variables on the EE of $S G$-NCTD-LIP. The results presented in Fig. 4A identified the highest $\mathrm{EE}$ at $0.24 \%$ phospholipid concentration. However, excessively high phospholipid resulted in the aggregation of phospholipids and decreased EE. It was revealed that the EE of SG-NCTD-LIP was lowered from 25 to $16 \%$ when increasing the amount of NCTD; the highest EE was observed for a 1:20 NCTD-phospholipid ratio (Fig. 4B). Cholesterol is a lipid used to improve liposome stability in vitro and in vivo (40). The results indicated that the highest EE was achieved at a 1:5 cholesterol-phospholipid mass ratio (Fig. 4C). At higher ratios, cholesterol may induce rapid vesicle aggregation. Its rigidity restricts the lipid bilayer flexibility and prevents NCTD accumulation inside the vesicle. The optimum incubation temperature was determined to be $40^{\circ} \mathrm{C}$ (Fig. 4D).

Optimization of the SG-NCTD-LIP formulation by orthogonal design. Four factors, NCTD-phospholipid mass ratio (factor A), phospholipid concentration (factor B), incubation temperature (factor C) and cholesterol-phospholipid mass ratio (factor D) were selected as main influencing factors. Each factor was studied at three levels and the EE was selected as the investigated indicator of the formulation. The orthogonal factors and levels are presented in Table I. Nine formulations were tested, according to the L9 $\left(3^{4}\right)$ orthogonal table. Table II displays the orthogonal test results and the variance analysis is presented in Table III. According to the extremum values (R) in Table II, the importance of the factors may be ranked as $A>B>C>D$. The optimum formulation composition was identified as 

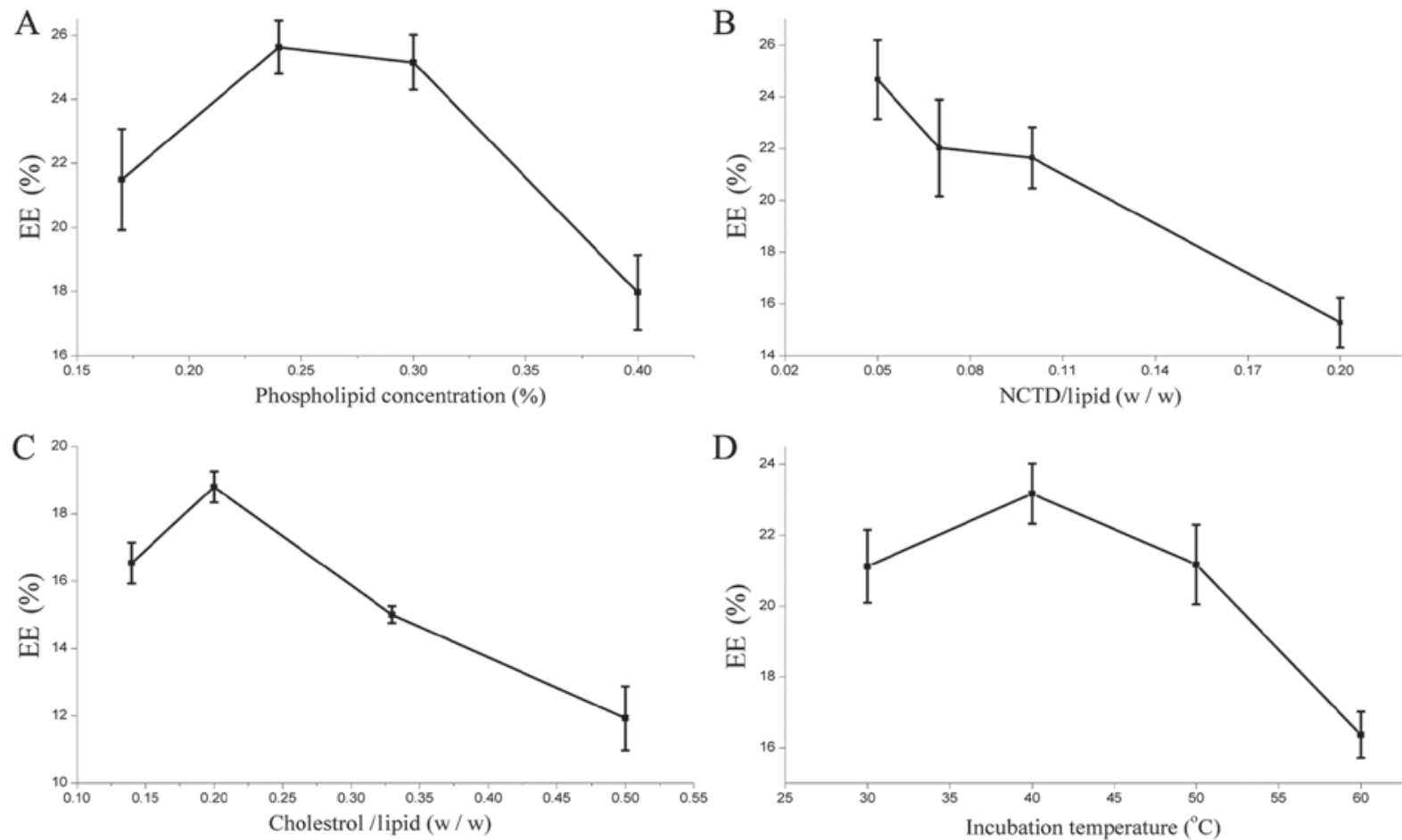

Figure 4. Effect of different preparation variables on the EE of SG-NCTD-LIP. Effect of changes in (A) phospholipid concentration (static parameters: 1:5 NCTD-phospholipid mass ratio and 1:7 cholesterol-phospholipid mass ratio at an incubation temperature of $50^{\circ} \mathrm{C}$ ), (B) NCTD-phospholipid mass ratio (static parameters: $0.24 \%$ Phospholipid concentration, 1:7 cholesterol-phospholipid mass ratio at an incubation temperature of $\left.50^{\circ} \mathrm{C}\right)$, (C) cholesterol-phospholipid mass ratio (static parameters: $0.24 \%$ Phospholipid concentration, 1:20 NCTD-phospholipid mass ratio at an incubation temperature of $50^{\circ} \mathrm{C}$ ) and (D) incubation temperature (static parameters: $0.24 \%$ Phospholipid concentration, 1:20 NCTD-phospholipid mass ratio and 1:5 cholesterol-phospholipid mass ratio) on EE. EE, encapsulation efficiency; NCTD, norcantharidin.

A1B1C2D3, with 1:5 NCTD-phospholipid mass ratio, 0.4\% phospholipid and 1:7 cholesterol-phospholipid mass ratio at an incubation temperature of $50^{\circ} \mathrm{C}$. The analysis of variance indicated that factors $\mathrm{A}, \mathrm{B}$ and $\mathrm{C}$ had a significant impact on the experimental outcome (Table III). According to the F-value, the impact of the factors may be scored as $\mathrm{A}>\mathrm{B}>\mathrm{C}>\mathrm{D}$, which supports earlier findings. Preparation and analysis of the optimal formulation mentioned above were repeated three times and the EE was $27.80 \pm 2.18 \%$. Prior to optimization of the NCTD-LIP formulation, literature was consulted (41-44) and combined with preliminary experiments, which investigated the effect of different masses of SG on the size, morphology, EE and stability of liposomes. Following this, $0.04 \%$ SG was identified as an appropriate amount of drug to be added, without affecting the formulation parameters of the liposome.

SG-NCTD-LIP size and morphology. Nanoparticles, between $70-200 \mathrm{~nm}$, function as drug delivery systems and are usually in the circulation for longer than free drugs $(45,46)$. In addition, such nanoparticles escape RES clearance, while larger particles $(>200 \mathrm{~nm}$ ) may be removed by the system. A small particle size $(<200 \mathrm{~nm})$, below the pore size of leaky vasculatures, along with poor lymphatic drainage may provide a suitable condition for the accumulation and localization in the tumor (47). The average particle size at the optimal formulation after 3 months of storage at $4^{\circ} \mathrm{C}$ was $87.5 \mathrm{~nm}$ with a PDI of 0.151 (Fig. 5). The morphological character of SG-NCTD-LIPs was evaluated by TEM. Liposomes were spherical, no aggregation or fusion occurred and the size was $\sim 100 \mathrm{~nm}$.
Stability of liposomes SG-NCTD-LIPS. Particle size and EE were evaluated following 3 months of storage at $4^{\circ} \mathrm{C}$ to study stability. Initially, the average particle size was $87.5 \mathrm{~nm}$ and $\mathrm{EE}$ was $27.80 \%$. There were no significant changes in the average particle size and EE following 3 months at $4^{\circ} \mathrm{C}$, the average particle size was $82.9 \mathrm{~nm}$ and EE was $27.28 \%$. The SG-NCTD-LIP stability was attributed to the presence of cholesterol, which increased the rigidity of the liposomal membrane and reduced permeability (48). The results demonstrated that SG-NCTD-LIPs were stable at $4^{\circ} \mathrm{C}$ for 3 months. However, the biological efficacy of SG-NCTD-LIPs following storage was not evaluated.

In vitro release of $N C T D$ from $S G$-NCTD-LIP. The in vitro release rate evaluation describes an essential step for drug development and quality control, which may reflect on the in vivo drug performance. $Q$ is used to denote the cumulative release percentage. The release rate of NCTD from SG-NCTD-LIP was significantly decreased compared with the free NCTD solution 1.5-12 $\mathrm{h}$ after incubation (Fig. 6). SG-NCTD-LIP may prolong the release of NCTD from liposomes. The release process of NCTD from SG-NCTD-LIP may be divided into two phases: In the first $4 \mathrm{~h}$, the release rate was high, with $65 \%$ of the total dose released. Unencapsulated NCTD and NCTD coordinating to the surface of the lipid bilayer may be responsible for the rapid release. This phase was defined as a rapid release phase. It was followed by a steady release, known as the slow release phase. To investigate the release properties of SG-NCTD-LIP, release data were fitted using various release models, including zero-order, first-order, Higuchi (49) and Weibull (50) kinetic models, and the 
Table I. Factors and levels for optimization using orthogonal design.

\begin{tabular}{lllcc}
\hline & \multicolumn{4}{c}{ Factor } \\
\cline { 2 - 5 } Level & $\mathrm{A}$ & $\mathrm{B}(\%)$ & $\mathrm{C}\left({ }^{\circ} \mathrm{C}\right)$ & $\mathrm{D}$ \\
\hline 1 & $1: 5$ & 0.040 & 40 & $1: 3$ \\
2 & $1: 15$ & 0.025 & 50 & $1: 5$ \\
3 & $1: 30$ & 0.015 & 60 & $1: 7$ \\
\hline
\end{tabular}

A, NCTD-phospholipid mass ratio; B, phospholipid concentration $\mathrm{C}$, incubation temperature; $\mathrm{D}$, cholesterol-phospholipid mass ratio.

Table II. Results of orthogonal testing.

\begin{tabular}{lrrrrr}
\hline No. & \multicolumn{1}{c}{$\mathrm{A}$} & $\mathrm{B}(\%)$ & $\mathrm{C}\left({ }^{\circ} \mathrm{C}\right)$ & $\mathrm{D}$ & $\mathrm{EE}(\%)$ \\
\hline 1 & 1.00 & 1.00 & 1.00 & 1.00 & 25.88 \\
2 & 1.00 & 2.00 & 2.00 & 2.00 & 23.40 \\
3 & 1.00 & 3.00 & 3.00 & 3.00 & 22.65 \\
4 & 2.00 & 1.00 & 2.00 & 3.00 & 27.63 \\
5 & 2.00 & 2.00 & 3.00 & 1.00 & 20.26 \\
6 & 2.00 & 3.00 & 1.00 & 2.00 & 23.96 \\
7 & 3.00 & 1.00 & 3.00 & 2.00 & 17.20 \\
8 & 3.00 & 2.00 & 1.00 & 3.00 & 15.53 \\
9 & 3.00 & 3.00 & 2.00 & 1.00 & 17.72 \\
$K 1$ & 71.93 & 70.71 & 65.37 & 63.86 & N/A \\
$K 2$ & 71.85 & 59.19 & 68.75 & 64.56 & N/A \\
$K 3$ & 50.45 & 64.33 & 60.11 & 65.81 & N/A \\
$R$ & 21.48 & 11.52 & 8.64 & 1.95 & N/A \\
\hline
\end{tabular}

A, NCTD-phospholipid mass ratio; B, phospholipid concentration; C, incubation temperature; $\mathrm{D}$, cholesterol-phospholipid mass ratio; $\mathrm{EE}$, encapsulation efficiency; $K_{n}$, sum of EE values at level $n ; R=K_{\max }-K_{\min }$.

optimal fit was determined by correlation coefficient. The fitting equations of the release curves are presented in Table IV. The results indicated that the release from SG-NCTD-LIP follows a first order model.

In vitro cytotoxicity study. The cytotoxicity of SG-LIP, free NCTD, NCTD-LIP and SG-NCTD-LIP towards HepG2 cells was evaluated using the MTT cytotoxicity assay. SG-LIP was assessed as a control and the concentration of SG in SG-LIP was equal to its concentration in SG-NCTD-LIP. Following incubation for $48 \mathrm{~h}$, an IR value of $\sim 6.35 \%$ was determined for SG-LIP, indicating no significant cytotoxicity against HepG2 cells (data not shown). The inhibitory effect of the samples increased with the NCTD concentration; all treatments revealed dose-dependent cytotoxic activity (Fig. 7). NCTD-LIP and SG-NCTD-LIP demonstrated significantly increased toxicity compared with free NCTD. In addition, when NCTD concentration was 5, 10 and $20 \mu \mathrm{g} / \mathrm{ml}$, SG-NCTD-LIP significantly increased cytotoxicity compared with NCTD-LIP after $48 \mathrm{~h}$ incubation. The $\mathrm{IC}_{50}$ of SG-NCTD-LIP, NCTD-LIP and free NCTD were
Table III. Variance analysis.

\begin{tabular}{lrrrcc}
\hline Source & \multicolumn{1}{c}{$S$} & $f$ & $M S$ & $F$-statistic & P-value \\
\hline A & 102.15 & 2 & 51.08 & 157.02 & $<0.01$ \\
B & 22.20 & 2 & 11.10 & 34.13 & $<0.05$ \\
C & 12.64 & 2 & 6.32 & 19.43 & $<0.05$ \\
D & 0.65 & 2 & 0.33 & 1.00 & $>0.05$ \\
Error & 0.65 & 2 & 0.33 & N/A & N/A \\
\hline
\end{tabular}

$S$, sum of deviation square; $f$, degree of freedom; $M S$, mean square; A, NCTD-phospholipid mass ratio; B, phospholipid concentration; $\mathrm{C}$, incubation temperature; $\mathrm{D}$, cholesterol-phospholipid mass ratio.

Table IV. Release curve-fitting equations of norcantharidin loaded liposomes modified with stearyl glycyrrhetinate.

\begin{tabular}{llc}
\hline Model & \multicolumn{1}{c}{ Fitting equation } & $R$ \\
\hline Zero-order & $Q=0.0972 t+0.2303$ & 0.9190 \\
First order & $\ln (1-Q)=-0.3336 t-0.2110$ & 0.9902 \\
Higuchi & $Q=0.3732 t^{1 / 2}+0.0350$ & 0.9764 \\
Weibull & $\ln (1 / 1-Q)=0.6040 \ln t+0.7481$ & 0.9561 \\
\hline
\end{tabular}

$R$, correlation coefficient; $Q$, accumulative release rate; $t$, time.

16.93, 24.03 and $49.79 \mu \mathrm{g} / \mathrm{ml}$, respectively. SG-NCTD-LIP was 1.42-fold more cytotoxic compared with NCTD-LIP. SG modification increased the cytotoxicity of the liposomes, which may be associated with the interaction between SG exposed on the liposome surface and GA receptors on the cell membrane.

Conclusions. SG-NCTD-LIP was successfully prepared using the ethanol injection method and the determined properties, including particle size, EE, release profile and stability, may meet the potential requirements of liver cancer therapy based on the in vitro experiments, which overcome the limitations of conventional chemotherapy by improving the bioavailability and stability of the drug molecules, and minimizing side effects by site-specific and targeted delivery of the drugs (51-53). The in vitro cytotoxicity study confirmed that SG modification of NCTD-LIP enhanced the inhibitory effects on hepatoblastoma cells. Therefore, SG-NCTD-LIP may be a potential drug delivery system for NCTD-targeted liver cancer therapy. Future experiments may include cytotoxicity studies against HepG2 cells compared with L02 normal liver cells, to confirm the safety and efficacy of SG-NCTD-LIP, further uptake studies and in vivo experiments.

\section{Acknowledgements}

Not applicable.

\section{Funding}

The present study was funded by grants from the National Natural Science Foundation of China (grant no. 81560653) and 

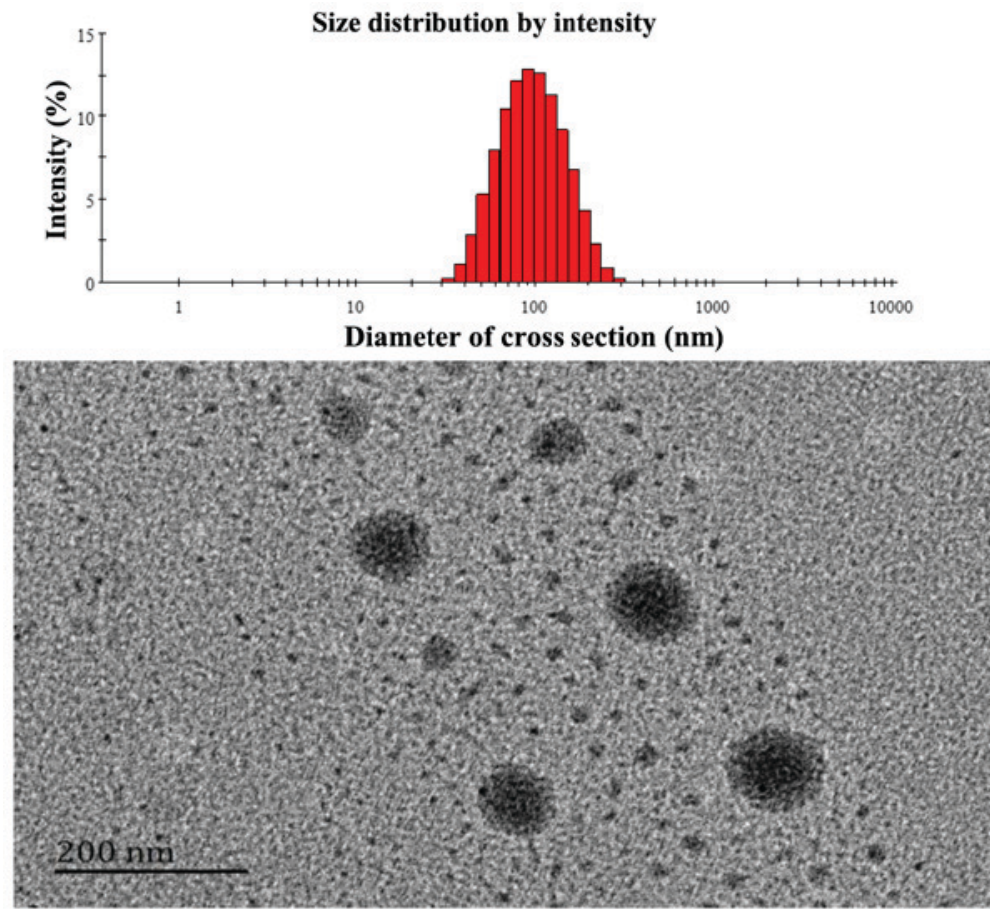

Figure 5. Particle size distribution (intensity) and TEM image of norcantharidin-loaded liposomes modified with stearyl glycyrrhetinate. TEM, transmission electron microscopy.

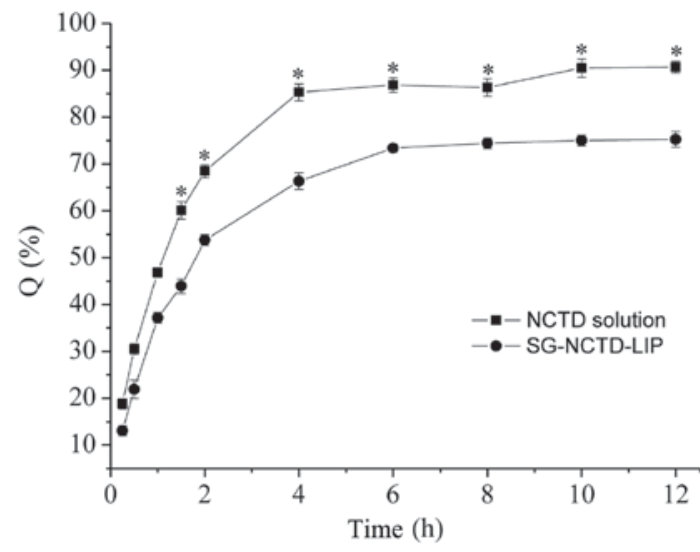

Figure 6. In vitro release of NCTD from solution and SG-NCTD-LIP over time. NCTD, norcantharidin; SG-NCTD-LIP, NCTD-loaded liposomes modified with stearyl glycyrrhetinate; $\mathrm{Q}$, cumulative release percentage. ${ }^{*} \mathrm{P}<0.05$ vs. SG-NCTD-LIP.

the Natural Science Foundation of Guangxi Province of China (grant no. 2016GXNSFAA380081).

\section{Availability of data and materials}

The datasets generated and analyzed during the current study are not publicly available due to the patent application but are available from the corresponding author on reasonable request.

\section{Authors' contributions}

WW was involved in the design of the study. JZ and WZ performed all experiments. DW performed the in vitro cytotoxicity studies. SL performed statistical analyses. All authors

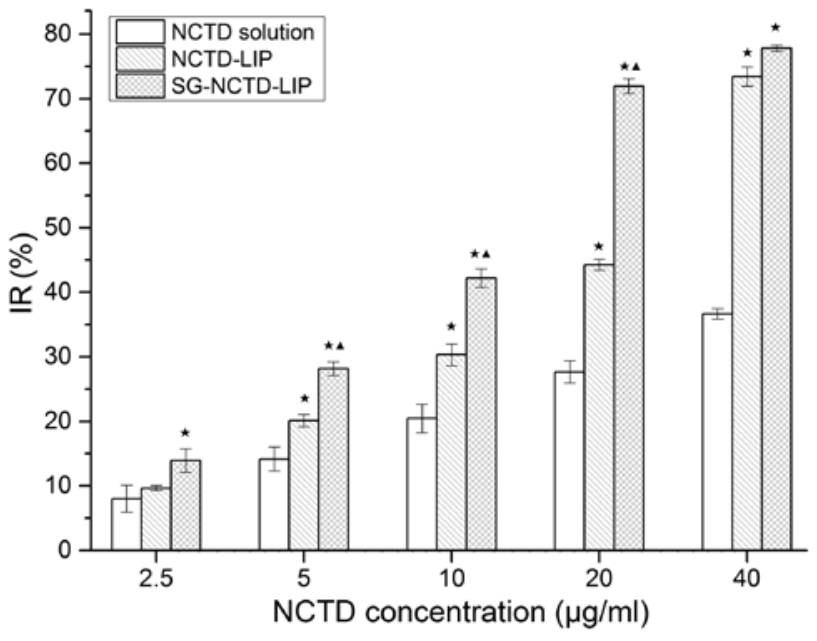

Figure 7. Inhibitory effects of different concentrations of NCTD in solution, NCTD-LIP and SG-NCTD-LIP on HepG2 cells after $48 \mathrm{~h} .{ }^{\star} \mathrm{P}<0.05$ vs. NCTD solution; ${ }^{\wedge} \mathrm{P}<0.05$ vs. NCTD-LIP. NCTD, norcantharidin; LIP, liposome; NCTD-LIP, NCTD-loaded liposomes, SG-NCTD-LIP, NCTD-loaded LIPs modified with stearyl glycyrrhetinate; IR, inhibition ratio.

participated in manuscript preparation and involved in the discussion of the results. All authors have read and approved the final manuscript.

\section{Ethics approval and consent to participate}

Not applicable.

\section{Patient consent for publication}

Not applicable. 


\section{Competing interests}

The authors declare that they have no competing interests.

\section{References}

1. Bei YY, Chen XY, Liu Y, Xu JY, Wang WJ, Gu ZL, Xing KL, Zhu AJ, Chen WL, Shi LS, et al: Novel norcantharidin-loaded liver targeting chitosan nanoparticles to enhance intestinal absorption. Int J Nanomedicine 7: 1819-1827, 2012.

2. Chen YC, Chang SC, Wu MH, Chuang KA, Wu JY, Tsai WJ and Kuo YC: Norcantharidin reducedcyclins and cytokines production in human peripheral blood mononuclearcells. Life Sci 84 218-226, 2009.

3. Wei CM, Wang BJ, Ma Y, Sun ZP, Li XL and Guo RC: Pharmacokinetics and biodistribution of $3 \mathrm{H}$-norcantharidin in mice. Yao Xue Xue Bao 42: 516-519, 2007 (In Chinese).

4. Chen YN, Chen JC, Yin SC, Wang GS, Tsauer W, Hsu SF and Hsu SL: Effect ormechanisms of norcantharidin-induced mitotic arrest and apoptosis inhuman hepatoma cells. Int J Cancer 100: $158-165,2002$.

5. McCluskey A and Sakoff JA: Small molecule inhibitors of serine/threonine protein phosphatases. Mini Rev Med Chem 1: 43-55, 2001.

6. Zheng LC, Yang MD, Kuo CL, Lin CH, Fan MJ, Chou YC, Lu HF, Huang WW, Peng SF and Chung JG: Norcantharidin-induced apoptosis of ags humangastric cancer cells throughreactive oxygen species production, and caspase- and mitochondria-dependent signaling pathways. Anticancer Res 36: 6031-6042, 2016.

7. Zhang S, Li G, Ma X, Wang Y, Liu G, Feng L, Zhao Y, Zhang G, Wu Y, Ye X, et al: Norcantharidin enhances ABT-737-induced apoptosis in hepatocellular carcinoma cells by transcriptional repression of Mcl-1. Cell Signal 24: 1803-1809, 2012.

8. Wan XY, Zhai XF, Jiang YP, Han T, Zhang QY and Xin HL: Antimetastatic effects of norcantharidin on hepatocellular carcinoma cells by up-regulating FAM46C expression. Am J Trans Res 9: 155-166, 2017.

9. Xiong X, Wu M, Zhang H, Li J, Lu B, Guo Y, Zhou T, Guo H, Peng R, Li X, et al: Atg5 siRNA inhibits autophagy and enhances norcantharidin-induced apoptosis in hepatocellular carcinoma. Int J Oncol 47: 1321-1328, 2015.

10. Lin X, Zhang B, Zhang K, Zhang Y, Wang J, Qi N, Yang S, He H and Tang X: Preclinical evaluations of norcantharidin-loaded intravenous lipid microspheres with low toxicity. Expert Opin Drug Deliv 9: 1449-1462, 2012.

11. Xie J, Zhang Y, Hu X, Lv R, Xiao D, Jiang L and Bao Q: Norcantharidin inhibits Wnt signal pathway via promoter demethylation of WIF-1 in human non-small cell lung cancer Med Oncol 32: 145, 2015

12. Liu MC, Liu L, Wang XR, Shuai WP, Hu Y, Han M and Gao JQ: Folate receptor-targeted liposomes loaded with a diacid metabolite of norcantharidin enhance antitumor potency for $\mathrm{H} 22$ hepatocellular carcinoma both in vitro and in vivo. Int $\mathrm{J}$ Nanomedicine 11: 1395-1412, 2016

13. Zeng Q and Sun M: Poly(lactide-co-glycolide) nanoparticles as carriers for norcantharidin. Materials Science and Engineering: C 29: 708-713, 2009

14. Ding XY, Hong CJ, Liu Y, Gu ZL, Xing KL, Zhu AJ, Chen WL, Shi LS, Zhang XN and Zhang Q: Pharmacokinetics, tissue distribution, and metabolites of a polyvinylpyrrolidone-coated norcantharidin chitosan nanoparticle formulation in rats and mice, using LC-MS/MS. Int J Nanomedicine 7: 1723-1735, 2012.

15. Yan D, Ni LK, Chen HL, Chen LC, Chen YH and Cheng CC: Amphiphilic nanoparticles of resveratrol-norcantharidin to enhance the toxicity in zebrafish embryo. Bioorg Med Chem Lett 26: 774-777, 2016.

16. Ding $X Y$, Hong $C J$ and Zhou $X$ : Mechanism of Polyvinylpyrrolidone-coated norcantharidin chitosan nanoparticle. Current Nanoscience 9: 401-406, 2013.

17. Torchilin VP: Recent advances with liposomes as pharmaceutical carriers. Nat Rev Drug Discov 4: 145-160, 2005.

18. Bangham AD, Standish MM and Watkins JC: Diffusion of univalent ions across the lamellae of swollen phospholipids J Mol Biol 13: 238-252, 1965.

19. Irie T, Watarai $S$ and Kodama H: Humoral immune response of carp (Cyprinus carpio) induced by oral immunization with liposome-entrapped antigen. Dev Comp Immunol 27: 413-421, 2003.
20. Kirby C, Clarke J and Gregoriadis G: Effect of the cholesterol content of small unilamellar liposomes on their Stability in vivo and in vitro. Biochem J 186: 591-598, 1980.

21. Zhang L, Gu FX, Chan JM, Wang AZ, Langer RS and Farokhzad OC: Nanoparticles in medicine: Therapeutic applications and developments. Clin Pharmacol Ther 83: 761-769, 2008

22. Ara MN, Matsuda T, Hyodo M, Sakurai Y, Hatakeyama H, Ohga N, Hida K and Harashima H: An aptamer ligand based liposomal nanocarrier system that targets tumor endothelial cells. Biomaterials 35: 7110-7120, 2014.

23. Kang H, O'Donoghue MB, Liu H and Tan W: A liposome-based nanostructure for aptamer directed delivery. Chem Commun (Camb) 46: 249-251,2010

24. Moghimi SM, Hunter AC and Murray JC: Long-circulating andtarget-specific nanoparticles: Theory to practice. Pharmacol Rev 53: 283-318, 2001

25. Maeda H: Tumor-selective delivery of macromolecular drugs via the EPReffect: Background and future prospects. Bioconjug Chem 21: 797-802, 2010.

26. Kibria G, Hatakeyama H, Ohga N, Hida K and Harashima $H$ Dual-ligand modification of PEGylated liposomes shows better cell selectivity and efficient gene delivery. J Control Release 153: 141-148, 2011.

27. Darvishi B, Manoochehri S, Esfandyari-Manesh M, Samadi N, Amini M, Atyabi F and Dinarvand R: Enhanced cellular cytotoxicity and antibacterial activity of $18-\beta$-Glycyrrhetinic Acidby Albumin-conjugated PLGA Nanoparticles. Drug Res 65: 617-623, 2015.

28. Kuang P, Zhao W, Su W, Zhang Z, Zhang L, Liu J, Ren G and Wang X: $18 \beta$-glycyrrhetinic acid inhibits hepatocellular carcinoma development by reversing hepatic stellate cell-mediated immunosuppression in mice. IntJ Cancer 132: 1831-1841, 2013.

29. Manns MP, Wedemeyer H, Singer A, Khomutjanskaja N, Dienes HP, Roskams T, Goldin R, Hehnke U and Inoue H; European SNMC Study Group: Glycyrrhizin in patients who failed previous interferon alpha-basedtherapies: Biochemical and histological effects after 52 weeks. J Viral Hepat 19: 537-546, 2012.

30. Tang ZH, Li T, Chang LL, Zhu H, Tong YG, Chen XP, Wang YT and Lu JJ: Glycyrrhetinic Acid triggers a protectiveautophagy by activation of extracellular regulated protein kinases in hepatocellular carcinoma cells. J Agric Food Chem 62: 11910-11916, 2014.

31. Irie A, Fukui $T$, Negishi $M$, Nagata $N$ and Ichikawa $A$ Glycyrrhetinic acid bound to 11 beta-hydroxysteroid dehydrogenase in rat liver microsomes. Biochim Biophys Acta 1160 229-234, 1992

32. Negishi $\mathrm{M}$, Irie $\mathrm{A}$, Nagata $\mathrm{N}$ and Ichikawa $\mathrm{A}$ : Specific binding of glycyrrhetinic acid to the rat liver membrane. Biochim Biophys Acta 1066: 77-82, 1991.

33. Tian Q, Zhang CN, Wang XH, Wang W, Huang W, Cha RT, Wang CH, Yuan Z, Liu M, Wan HY and Tang H: Glycyrrhetinic acid-modified chitosan/poly (ethylene glycol) nanoparticles for liver-targeted delivery. Biomaterials 31: 4748-4756, 2010.

34. Tian Q, Wang XH, Wang W, Zhang CN, Wang P and Yuan Z: Self-assembly and liver targeting of sulfated chitosan nanoparticles functionalized with glycyrrhetinic acid. Nanomedicine 8 870-879, 2012.

35. Zhang J, Zhang M, Ji J, Fang X, Pan X, Wang Y, Wu C and Chen M: Glycyrrhetinic acid-mediated polymeric drug delivery targeting the acidic microenvironment of hepatocellular carcinoma. Pharm Res 32: 3376-3390, 2015

36. Chintharlapalli S, Papineni S, Jutooru I, McAlees A and Safe S: Structure-dependent activity of glycyrrhetinic acid derivatives asperoxisome proliferator-activated receptor \{gamma\} agonists in colon cancer cells. Mol Cancer Ther 6: 1588-1598, 2007.

37. Marzban E, Alavizadeh SH, Ghiadi M, Khoshangosht M, Khashayarmanesh Z, Abbasi A and Jaafari MR: Optimizing the therapeutic efficacy of cisplatin PEGylated liposomes via incorporation of different DPPG ratios: In vitro and in vivo studies. Colloids Surf B Biointerfaces 136: 885-891, 2015.

38. Chi L, Na MH, Jung HK, Vadevoo SM, Kim CW, Padmanaban G, Park TI, Park JY, Hwang I, Park KU, et al: Enhanced delivery of liposomes to lung tumor through targeting interleukin-4 receptor on both tumor cells and tumor endothelial cells. J Control Release 209: 327-336, 2015

39. Liu D, Xing J, Xiong F, Yang F and Gu N: Preparation and in vivo safety evaluations of antileukemichomoharringtonine-loaded PEGylated liposomes. Drug Dev Ind Pharm 43: 652-660, 2017.

40. Kirby C, Clarke J and Gregoriadis G: Effect of the cholesterol content of small unilamellar liposomeson their stability in vivo and in vitro. Biochem J 186: 591-598, 1980 
41. Li J, Xu H, Ke X and Tian J: The anti-tumor performance of docetaxel liposomes surface-modified with glycyrrhetinic acid. J Drug Targeting 20: 467-473, 2012.

42. Chen J, Jiang H, Wu Y, Li Y and Gao Y: A novel glycyrrhetinic acid-modified oxaliplatin liposome for liver-targeting and in vitro/vivo evaluation. Drug Des Dev Ther 9: 2265-2275, 2015.

43. Cheng M, Gao X, Wang Y, Chen H, He B, Xu H, Li Y, Han J and Zhang Z: Synthesis of glycyrrhetinic acid-modified chitosan5-fluorouracil nanoparticles and its inhibition of liver cancercharacteristics in vitro and in vivo. Mar Drugs 11: 3517-3536, 2013.

44. Tian Q, Wang XH, Wang W, Zhang CN, Wang P and Yuan Z: Self-assembly and liver targeting of sulfated chitosannanoparticles functionalized with glycyrrhetinic acid. Nanomedicine 8: 870-879, 2012

45. Ossipov DA: Nanostructured hyaluronic acid-based materials for active delivery tocancer. Expert Opin Drug Deliv 7: 681-703, 2010.

46. Yang T, Choi MK, Cui FD, Kim JS, Chung SJ, Shim CK and Kim DD: Preparationand evaluation of paclitaxel-loaded PEGylated immunoliposome. J Control Release 120: 169-177, 2007.

47. Ravar F, Saadat E, Gholami M, Dehghankelishadi P, Mahdavi M, Azami S and Dorkoosh FA: Hyaluronic acid-coated liposomes for targeted delivery of paclitaxel, in-vitro characterization and in-vivo evaluation. J Control Release 229: 10-22, 2016.
48. Fan MH, Xu SY, Xia SQ and Zhang XM: Preparation of salidroside nano-liposomes by ethanol injection method and in vitro release study. Eur Food Res Technol 227: 167-174, 2008.

49. Higuchi T: Mechanism of sustained-action medication. Theoretical analysis of rate of release of solid drugs dispersed in solid matrices. J Pharm Sci 52: 1145-1149, 1963.

50. Weibull W: A statistical distribution of wide applicability. J Appl Mech 18: 293-297, 1951

51. Dutta R and Mahato RI: Recent advances in hepatocellular carcinoma therapy. Pharmacol Ther 173: 106-117, 2017.

52. Sarfraz M, Afzal A, Raza SM, Bashir S, Madni A, Khan MW, Ma X and Xiang G: Liposomal co-delivered oleanolic acid attenuates doxorubicin-induced multi-organ toxicity in hepatocellular carcinoma. Oncotarget 8: 47136-47153, 2017.

53. Himanshu P, Radha R and Vishnu A: Liposome and their applications in cancer therapy. Braz Arch Boil Technol 59: e16150477, 2016.

(i) This work is licensed under a Creative Commons

Attribution-NonCommercial-NoDerivatives 4.0 International (CC BY-NC-ND 4.0) License. 